Pteridines

Vol. 11, 2000, pp. $60-63$

\title{
Dihydroneopterin and the generation of superoxide from iron ions
}

\author{
Karl Oettl, Joachim Greilberger and Gilbert Reibnegger \\ Institute for Medical Chemisty and Pregl-Laboratory, Karl-Franzens-University Graz, Harrachgasse 21/II, A- \\ 8010 Graz, Austria
}

This work was financially supported by the Austrian "Fonds zur Förderung der Wissenschaftlichen Forschung". project P12366-CHE.

\begin{abstract}
We describe the impact of dihydroneopterin on the formation of superoxide radicals. As radical source ferrous iron in air-saturated aequeous solution was used. Detection of superoxide was achieved using reduction of nitroblue tetrazolium to formazan. Dihydroneopterin decreases formazan formation when high concentrations of ferrous iron are used which induce a rapid superoxide formation within minutes. On the other hand, when low concentrations of ferrous iron are used, addition of dihydroneopterin to the assay mixture leads to an increase ir. formazan formation for several hours of incubation. We therefore conclude that the radical promoting activity 0 : dihydroneopterin is physiologically more relevant compared to its radical scavenging properties.
\end{abstract}

Key words: oxygen species, nitroblue tetrazolium, formazan, iron.

\section{Introduction}

Pterins have been reported to interact with different radical-mediated reactions in chemical, biochemical and biological systems (1-16). Aromatic pterins were discussed to have radical promoting activity, or radical scavenging activity, or not to interfere with free radicals. On the other hand, reduced pterins were shown to be radical scavengers by different authors. However, high concentrations of dihydroneopterin showed radical promoting activity $(8,10)$. Recently we found that in solutions of dihydroneopterin and other dihydropterins hydroxyl radicals are formed (15, 16). We showed that molecular oxygen which is reduced via superoxide, is the source of hydroxyl radicals, and iron ions play an important catalytical role in that system. While dihydroneopterin is responsible for the amount of hydroxyl radicals generated, iron ions determine the kinetics of the reaction. Until now only tetrahydropterins have been shown to form complexes with iron ions (17-19) but dihydroneopterin was found to reduce iron from the ferric to the ferrous form (20). With this background a connection of the iron reducing ability of dihydroneopterin and its radical promoting effect is obvious. Nevertheless, dihydroneopterin can be shown to have both, a radical promoting and radical scavenging effect in the presence of iron ions depending on the experimental conditions as we show in this study.

\section{Material and Methods}

\section{Reagents}

7,8-Dihydroneopterin was obtained from Dr. Schircks laboratory (Jona, Switzerland). Superoxide dismutase (EC 1.11.1.6), catalase, (EC 1.15.1.1), nitro blue tetrazolium (NBT), and 3-[N-morpholino]propanesulfonic acid (MOPS) were purchased from Sigma (Vienna, Austria). Phosphate buffered saline (PBS), consisting of $1.15 \mathrm{~g} / 1 \quad \mathrm{Na}_{2} \mathrm{HPO}_{4}, 0.2 \mathrm{~g}$ ! $\mathrm{KH}_{2} \mathrm{PO}_{4}, 0.1 \mathrm{~g} / 1 \mathrm{CaCl}_{2}, 0.1 \mathrm{~g} / 1 \mathrm{MgCl}_{2} \cdot 6 \mathrm{H}_{2} \mathrm{O}, 0.2 \mathrm{~g} 1$ $\mathrm{KCl}, 8 \mathrm{~g} / 1 \mathrm{NaCl}$, pH 7.4 was obtained by PAA (Linz. Austria). All other reagents were obtained from Merck (Darmstadt, Germany).

Superoxide formation

As a source of superoxide $\mathrm{FeSO}_{4}$ was used, the detection was performed using NBT which is reduced to a coloured formazan by the action of superoxide

\footnotetext{
Correspondence to: Dr. Karl Oettl, Institute for Medical Chemistry and Pregl-Laboratory, Harrachgasse 21/II, A-8010 Graz, Austria

Phone: ++433163807544, Fax: ++433163809610,E-mail: karl.oettl@kfunigraz.ac.at
} 
(21-23). Formazan formation is followed photometrically at $560 \mathrm{~nm}$. Two different sets of conditions were used: low iron concentrations (10 $\mu \mathrm{M})$ in the presence of EDTA in PBS or high iron concentrations $(100 \mu \mathrm{M})$ in MOPS buffer, both at $\mathrm{pH}$ 7.4. In the case of PBS $0.035 \%$ (w/v) Triton X-100 was added to the buffer. Formazan formation was followed up to $10 \mathrm{~min}$ in the case of high and up to 4 hours in the case of low iron concentration. Stock solutions of NBT and dihydroneopterin were prepared in the buffer used immediately before the assay. Solutions of ferrous sulfate were prepared in water deionized by a Milli-Q 185 apparatus (Millipore, Vienna, Austria).

\section{Results and discussion}

When low iron concentrations in PBS were used the conditions are similar to those applied earlier for measuring hydroxyl radical formation (15). Under these conditions iron autoxidation is favoured by the presence of both, EDTA and phosphate $(24,25)$. The formation of superoxide under these conditions up to 4 hours is shown in Fig. 1. When only EDTA chelated iron is present in the assay low formazan concentrations are produced during the first $30 \mathrm{~min}$. Thereafter the absorption remains constant during the incubation time.

In the case of dihydroneopterin without addition of iron ions formazan formation is continued throughout the assay and is higher compared to iron alone. When both, dihydroneopterin and chelated iron ions are present in the assay, superoxide is formed at the highest rate in a linear manner until the end of the incubation. Addition of $200 \mathrm{U} / \mathrm{ml}$ of catalase to the assay mixture shows almost no effect, while SOD $(200 \mathrm{U} / \mathrm{ml})$ decreases formazan formation to $17 \pm 6 \%$ (only dihydroneopterin) and $7 \pm 4 \%$ (dihydroneopterin + iron), respectively. If instead of ferrous iron ferric iron is used, the results are essentially the same.

We suppose that traces of iron ions in the buffer are responsible for superoxide formation which ceases after completed autoxidation of ferrous iron. Dihydroneopterin on the other hand is regenerating ferrous iron again and so prolonging superoxide formation. In this case both, reduction and autoxidation of iron have to take place simultanously in the presence of dihydroneopterin.

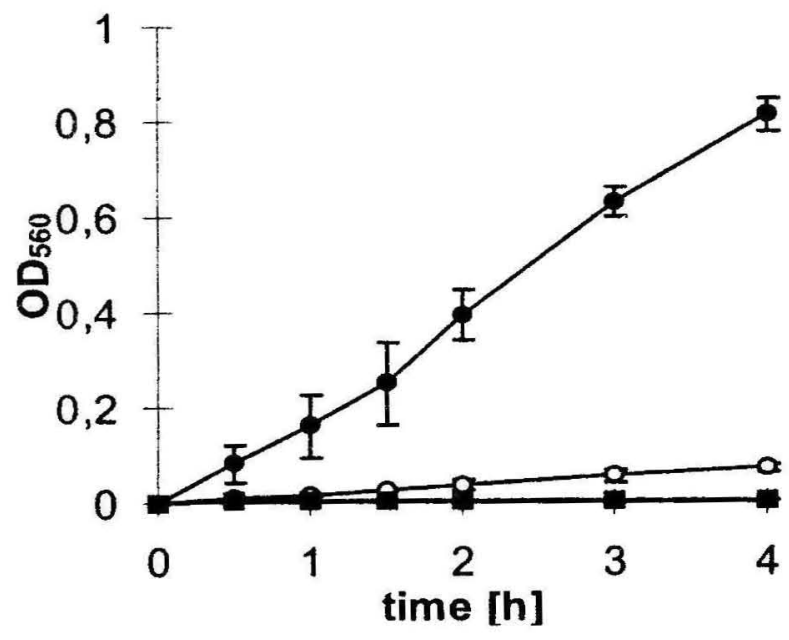

Fig. 1: Formation of formazan in PBS. NBT $(250 \mu \mathrm{M})$ was incubated in PBS, pH 7.4 in the presence of $0.035 \%$ (w/v) Triton X-100 and $10 / 20 \mu \mathrm{M} \mathrm{Fe}{ }^{2+} /$ EDTA $(\bullet), 500 \mu \mathrm{M}$ dihydroneopterin (O), or 10/20 $\mu \mathrm{M} \mathrm{Fe} \mathrm{FDTA}^{2+} / \mathrm{EDT}^{+}$ $500 \mu \mathrm{M}$ dihydroneopterin (๑). Formazan formation was followed photometrically. Mean values \pm S.D. of two experiments are shown.

When high concentrations of ferrous iron are utilized for superoxide formation autoxidation happens to fast for normal kinetic measurements in phosphate buffer. Therefore MOPS buffer was used in the absence of EDTA for the superoxide assays. Under these conditions dihydroneopterin has a different effect compared to the first set of experiments (Fig. 2). In the absence of dihydroneopterin superoxide is formed very rapidly after a short lag time. This lag time is necessary for the formation of the optimal ratio of ferrous to ferric iron as shown for the initiation of lipid peroxidation by iron ions $(26,27)$ and is suppressed by addition of ferric iron to the assay mixture (data not shown). Addition of dihydroneo- 
pterin results in a prolonged lag time, a slower formazan formation and a reduced maximum formazan formation. A similar effect was found when the iron concentration was decreased (data not shown). Dihydroneopterin leads to a lower availability of iron for autoxidation and therefore superoxide formation.

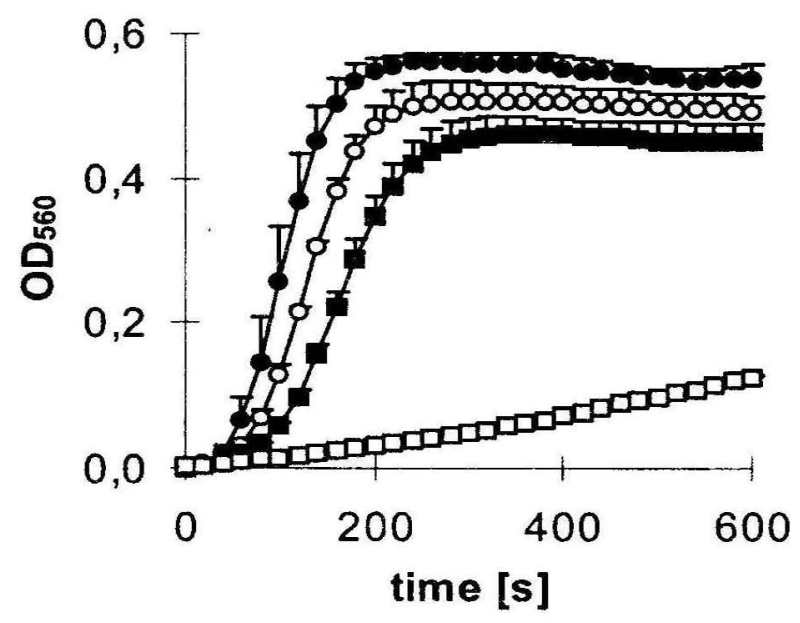

Fig. 2: Formation of formazan in MOPS. NBT $(250 \mu \mathrm{M})$ was incubated in MOPS buffer pH 7.4 in the presence of $100 \mu \mathrm{M}$ ferrous ions and increasing concentrations of dihydroneopterin: $0,(\bullet) ; 50(0), 100(*), 300(\square) \mu .1$ Mean values \pm S.D. of three experiments are shown.

The property of dihydroneopterin as a reducing agent results in an increased superoxide formation when iron is present at low concentrations, recycling ferric iron to the ferrous form. On the other hand, under conditions of high reaction rates of iron autoxidation superoxide formation is decreased. As normally iron ions are not present in blood under physiological conditions, the results obtained from the incubations with high iron concentrations are physiologically of minor relevance. However, low concentrations of iron may be liberated under conditions leading to activation of macrophages and secretion of dihydroneopterin (28). Moreover, other reducing agents have been shown to liberate iron ions from its protein bound form (29). If that is true for dihydroneopterin, too, it may support itself with iron ions and generate conditions leading to the formation of superoxide and hydroxyl radicals, as described earlier $(15,16)$.

From our data we conclude that one has to be aware that the experimental conditions chosen for testing a compound upon its radical promoting or scavenging properties are rather critical. Dihydroneopterin which was shown to act as radical scavenger as well as radical promoting agent may indeed serve as both even in similar incubation systems measuring solely formation of superoxide radicals. As free iron ions are physiologically not available and under pathological conditions only at low concentrations we conclude that dihydroneopterin under such conditions ma: primarily act as a prooxidant. For a detailed view. however, interactions with reactive nitrogen species have to be considered too and are under work at the moment.

\section{References}

1.Heales SJR., Blair J, A Meinschad C, Ziegler I Inhibition of monocyte luminol-dependent chemiluminescence by tetrahydrobiopterin, and the free radical oxidation of tetrahydrobiopterin, dihydrobiopterin and dihydroneopterin.

Cell Biochem Funct 1988; 6: 191-195.

2.Kojima S, Icho T, Kajiwara Y, Kubota K. Neopterin as an endogenous antioxidant. FEBS Lett 1992; 304: 163-166.

3. Weiss G, Fuchs D, Hausen, A, et al. Neopterin modulates toxicity mediated by reactive oxygen and chlorine species. FEBS Lett 1993; 321: 89-92.

4.Shen $\mathrm{R}$. Inhibition of luminol-enhanced chemiluminescence by reduced pterins. Arch Biochem Biophys 1994; 310: 60-63.

5. Kojima S, Ona S, Iizuka I, Arai T, Mori H, Kubota K. Antioxidative activity of 5,6,7,8-tetrahydrobiopterin and its inhibitory effect on paraquat-induced 
cell toxicity in cultured rat hepatocytes. Free Rad Res 1995; 23: 419-430.

6. Gieseg S, Reibnegger G, Wachter H, Esterbauer, $H$. 7,8-dihydroneopterin inhibits low density lipoprotein oxidation in vitro. Evidence that this macrophage secreted pteridine is an antioxidant. Free Rad Res 1995; 23:123-136.

7. Kojima S. Neopterin as an endogenous antioxidant. Pteridines 1995; 6: 181-184.

8. Baier-Bitterlich G, Fuchs D, Murr C et al. Effect of neopterin and 7,8-dihydroneopterin on tumor necrosis factor- $\alpha$ induced cell death, FEBS Lett 1995; 364: 234-238.

9. Reibnegger G, Fuchs D, Murr C, Dierich MP, Pfleiderer W, Wachter $H$. Effects of pteridines on luminol-dependent chemiluminescence induced by chloramine-T. Free Rad Biol Med 1995; 18: 515-523. 10. Murr C, Baier-Bitterlich G, Fuchs D, et al. Effects of neopterin derivatives on $\mathrm{H}_{2} \mathrm{O}_{2}$-induced luminol chemiluminescence: mechanistic aspects. Free Rad Biol Med 1996; $21: 449-456$.

11. Horejsi R, Estelberger W, Mlekusch, W, et al. Effects of pteridines on chloramine-T-induced growth inhibition in E.coli strains: correlations with molecular structure. Free Rad Biol Med 1996; 21: 133-138.

12. Oettl K, Dikalov S, Freisleben HJ, Mlekusch W, Reibnegger G. Spin trapping study of antioxidant properties of neopterin and 7,8-dihydroneopterin. Biochem Biophys Res Commun 1997; 234: 774-778.

13. Widner B, Baier-Bitterlich G, Wede I, Wirleitner B, Wachter H, Fuchs D. Neopterin: Indicator of oxidative stress and part of the cytotoxic armature of activated macrophages in humans. Pteridines 1998; 9 : 91-102.

14. Widner B, Baier-Bitterlich G, Wede I, Wirleitner, B, Fuchs D. Neopterin derivatives modulate the nitration of tyrosine by peroxynitrite. Biochchem Biophys Res Commun 1998; 248: 341-346.

15. Oettl K, Wirleitner B, Baier-Bitterlich G, Grammer T, Fuchs D, Reibnegger G. Formation of oxygen radicals in solutions of 7,8-dihydroneopterin. Biochem Biophys Res Commun 1999; 264: 262-267. Oettl K, Pfleiderer W, Reibnegger G. Formation of oxygen radicals in solutions of different 7,8dihydropterins: quantitative structure-activity relationships. Helv Chim Acta 2000; 83: 954-965
17. Schäfer A, Fischer B, Paul H, Bosshard R, He se M, Viscontini M. Electrospray-ionisation mass spectrometry: detection of a radical cation present in solution: new results on the chemistry of (tetrahydropteridinone)-metal complexes. Helv Chim Acta 1992; 75: 1955-1964.

18. Fischer $B$, Schäfer $A$, Bosshard $R$, Hesse $M$, Viscontini $M$. New results on the chemistry of metaltetrahydro-pterin complexes. Pteridines 1993; 4: 206209.

19. Fischer B, Schäfer A, Hegetschweiler $K$, Viscontini $M$. Iron and tetrahydropterin complex with 1,3,5-triamino-2,4,6-trihydroxycyclohexane as ligand. Pteridines 1995; 6: 87-89.

20. Hausen A, Fuchs D, Reibnegger G, Werner ER, Werner-Felmayer $\mathbf{G}$, Wachter $\mathrm{H}$. Reduction of ferric iron by 7,8-dihydroneopterin. Pteridines 1990; 2: 8385.

21. Beauchamp C, Fridovich I. Superoxide dismutase: improved assays and an assay applicable to acrylamide gels. Anal Biochem 1971; 44: 276-287.

22. Nishikimi $M$. The generation of superoxide anion in the reaction of tetrahydropteridines with molecular oxygen. Arch Biochem Biophys 1975; 166: 273-279.

23. Liochev SI, Batinic-Haberle I, Fridovich I. The effect of detergents on the reduction of tetrazolium salts. Arch Biochem Biophys 1995; 324: 48-52.

24. Aust SD, Morehouse LA, Craig ET. Role of metals in oxygen radical reactions. J Free Rad Biol Med 1985; 3-25.

25. Burkitt MJ, Gilbert BC. The autoxidatioin of iron(II) in aqueous systems: the effects of iron chelation by physiological, non-physiological and therapeutical chelators on the generation of reactive oxygen species and the inducement of biomolecular damage. Free Rad Res Comms 1991; 14: 107-123.

26. Bucher JR, Tien M, Aust SD. The requirement for ferric in the initiation of lipid peroxidation by chelated ferrous iron. Biochem Biophys Res Commun 1983; 111: 777-784.

27. Braughler JM, Duncan LA, Chase RL. The involvement of iron in lipid peroxidation. $\mathrm{J}$ Biol Chem 1986; 261: 10282-10289.

28. Ryan TP, Aust SD. The role of iron in oxygenmediated toxicities. Crit Rev Toxicol 1992; 22: 119 141.

29. Halliwell B, Gutteridge MC. Oxygen toxicity, oxygen radicals, transition metals and disease. Biochem J 1984; 219: 1-14. 